\title{
Coglueing Homotopy Equivalences
}

\author{
RoNALD BROWN and PHILIP R. HEATH
}

\section{Introduction}

Suppose given a commutative diagram of maps of topological spaces

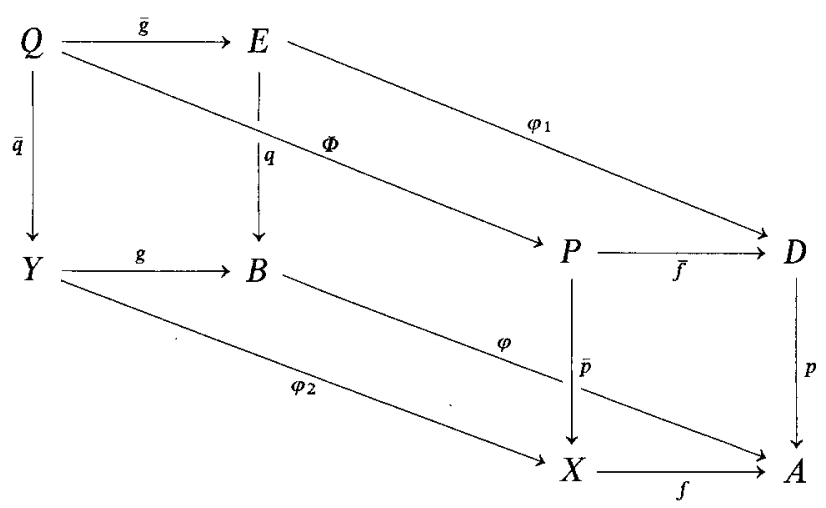

in which the front square is a pull-back. Then $P$ is often called the fibre-product of $f$ and $p$, and it is also said that $\bar{p}: P \rightarrow X$ is induced by $f$ from $p$. The map $\Phi: Q \rightarrow P$ is determined by $\varphi_{1}$ and $\varphi_{2}$. Our object is to give conditions on the front and back squares which ensure that if $\varphi_{1}, \varphi_{2}$ and $\varphi$ are homotopy equivalences, then so also is $\Phi$.

First of all we shall assume throughout that the back square of (1.1) as well as the front square, is a pull-back.

Second recall that a map $q: E \rightarrow B$ has the WCHP (weak covering homotopy property) if it has the covering property for all homotopies $Z \times I \rightarrow B$ which are stationary on $Z \times\left[0, \frac{1}{2}\right]$. This property has been shown by Dold [3] and Weinzweig [7] to be convenient for studying fiber homotopy equivalences, and our results will extend some of theirs.

For the rest of this section we will assume that in (1.1) $p$ and $q$ have the WCHP. Then our main object is the following theorem which will be proved in Sections 2 to 5.

(1.2) Theorem (The coglueing theorem). Under the above assumptions, if $\varphi, \varphi_{1}$, $\varphi_{2}$ are homotopy equivalences, then so also is $\Phi$.

We call this a "coglueing theorem " because its dual (in the sense of EckmannHilton) is very naturally called a "glueing theorem". Such a glueing theorem is proved in [1] and it seems to us that the coglueing and glueing theorems are so to speak the "first theorems" in the theory of fibrations and cofibrations 
respectively. The proof of Theorem 1.2 is based on the proof of the dual theorem $[1 ; 7.5 .7]$ but it is worth giving in detail firstly to make the exposition here self-contained, secondly in order to generalise to the WCHP (as against just the CHP), thirdly in order to present more consequences of the method than are given in [1] and fourthly, because the present theorem implies the dual theorem (but not conversely), and in slightly more generality, as is shown by Heath in [5a]. In particular we now give some corollaries of the coglueing theorem. Even these special cases have not, as far as we know, been published before.

First of all, let us write, as is common,

$$
Q=g^{*}(E), P=f^{*}(D)
$$

and take $Y=X, \varphi_{2}=1$. Then $f=\varphi g$ and so $P=(\varphi g)^{*}(D)=g^{*} \varphi^{*}(D)$.

(1.3) Corollary. If $\varphi: B \rightarrow A, \varphi_{1}: E \rightarrow D$ are homotopy equivalences, then so also is $\Phi: g^{*}(E) \rightarrow g^{*} \varphi^{*}(D)$.

Next replace (1.1) by the following diagram

and we obtain

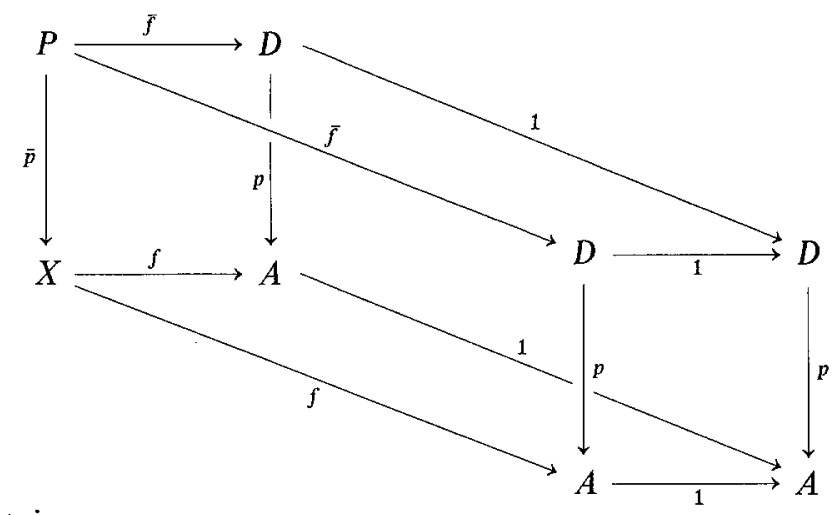

(1.4) Corollary. If $f: X \rightarrow A$ is a homotopy equivalence, then so also is $\bar{f}$ : $f^{*}(D) \rightarrow D$.

It follows from this Corollary and Theorem (3.4) that the pair $(\bar{f}, f)$ is a homotopy equivalence $\bar{p} \rightarrow p$ of maps.

For the next application we concentrate attention on the map of fibrations

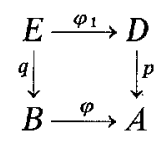

Let $x \in B$, and let $F_{x}=q^{-1}(x), F_{\varphi(x)}=p^{-1} \varphi(x)$ be the fibres of $q, p$ over $x, \varphi(x)$. respectively. Then $\varphi_{1}$ restricts to a map $F_{x} \rightarrow F_{\varphi(x)}$. Of course if in (1.1) we take $Y=\{x\}, X=\{\varphi(x)\}$ and let $g, f$ be the inclusions; then $Q, P$ can be identified with $F_{x}, F_{\varphi(x)}$ respectively, and $\Phi: Q \rightarrow P$ then becomes the restriction of $\varphi_{1}$. 
(1.5) Corollary. If $\varphi$ and $\varphi_{1}$ are homotopy equivalences, then so also is $\Phi$ : $F_{x} \rightarrow F_{\varphi(x)}$.

In $\S 6$ we apply Corollary (1.5) to prove that the Serre spectral sequence of a Serre fibration applies also to a map which has the WCHP.

In the case $F_{x}$ and $F_{\varphi(x)}$ are of the homotopy type of CW-complexes, Corollary (1.5) can be deduced from the exact homotopy sequence of a fibration, the 5-lemma, and Whitehead's theorem. Corollary (1.4) can be proved similarly if $D$ and $f^{*}(D)$ are of the homotopy type of CW-complexes. But such results are not as general as ours, and in any case do not seem to us to be in the spirit of the theory of fibrations.

Corollary (1.5) is also obvious in the category of maps of spaces with base point and homotopies rel base point (and if $x$ is the base point of $B$ ). In this theory, the CHP always means that homotopies rel base point lift to homotopies rel base point. But such a theory is not the obvious one for fibrations. In fact one of the difficulties of the Eckmann-Hilton duality is this restriction to spaces with base point, since in many practical cases one either wants no base point at all, or a free choice of base point.

For another application, consider the pull-back square

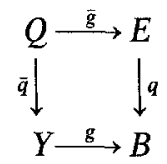

in which $q$ has the WCHP.

(1.6) Corollary. If $Y$ and $E$ are contractible, then $Q$ is either empty or is of the homotopy type of $\Omega(B, b)$, the space of loops on $B$ starting at some point $b \in B$.

Proof. If $g(Y) \cap q(E)$ is empty, then $Q$ is empty. Otherwise, let $b \in g(Y) \cap q(E)$, and consider the pull-back square

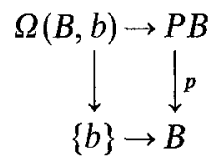

in which $p$ is the usual fibration of paths with initial point at $b$. Because $p$ is a fibration with contractible total space, $q: E \rightarrow B$ lifts to a map $\varphi_{1}: E \rightarrow P B$; also there is a unique map $\varphi_{2}: Y \rightarrow\{b\}$. Then $\varphi_{1}, \varphi_{2}$ are homotopy equivalences. By the coglueing theorem, the map $\Phi: Q \rightarrow \Omega(B, b)$ determined by $\varphi_{1}, \varphi_{2}$ and 1: $B \rightarrow B$, is a homotopy equivalence.

Related to the results of this paper is the fact that if $p$ has the WCHP then the homotopy type of $f^{*}(D)$ depends only on the homotopy class of $f[3$; Corollary 6.6]. However there is a strong case for expressing in the context of groupoid operations both this fact and some known kinds of operations; this will be done in a paper by Heath. 


\section{Elementary Definitions and Results}

For most of this paper, and unless mentioned otherwise, homotopies will be of length 1 , and the sum, or composite, of two homotopies $F: f \simeq g, G: g \simeq h$, will be written $G+F: f \simeq h$. A homotopy $F: Z \times I \rightarrow A$ is said to be bordered if there are numbers $r, s$ in ]0,1[ such that $r \leqq s$ and for all $z \in Z$

$$
\begin{array}{ll}
F(z, t)=F(z, 0) & \text { for } t \in[0 ; r], \\
F(z, t)=F(z, 1) & \text { for } t \in[s, 1] .
\end{array}
$$

These last conditions are expressed as: $F|Z \times[0, r], F| Z \times[s, 1]$ are stationary. Note that the sum of two bordered homotopies is bordered, and the reverse $-F$ of a bordered homotopy $F$ is bordered.

It is easily seen that a map $p: D \rightarrow A$ has the WCHP if and only if for any space $Z$ and map $f: Z \rightarrow D$, any bordered homotopy $F: Z \times I \rightarrow A$ of $p f$ lifts to a homotopy $H$ of $f$; further $H$ may be assumed to be bordered, though it will not necessarily be stationary on the same intervals as $F$. It is well-known $[3,7]$ that if $p: D \rightarrow A$ has the WCHP, and $f: X \rightarrow A$ is a map, then $\bar{p}: f^{*}(D) \rightarrow X$, the pull-back of $p$ by $f$, also has the WCHP.

Let $p: D \rightarrow A, u: Z \rightarrow A$ be maps. We are interested

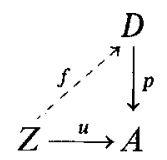

in maps $f: Z \rightarrow D$ which lift $u$ (i.e. which satisfy $p f=u$ ) and in the homotopy classes of these rel $p$. (Two lifts $f, g$ of $u$ are homotopic rel $p$ if there is a homotopy $F: f \simeq g$ such that $p F$ is stationary.) The set of these homotopy classes is written $Z, D \backslash u$.

The main object of this section is the following result, in which $\pi A^{Z}$ is the track groupoid as defined in [1], and $\mathscr{S e t}_{\text {et }}$ denotes the category of sets.

(2.1) Proposition. If $p: D \rightarrow A$ has the $W C H P$, then there is a functor $\Gamma: \pi A^{Z} \rightarrow$ Set such that on objects $\Gamma(u)=Z, D \backslash u$. Further, if $\alpha \in \pi A^{Z}(u, v)$ and the lifts $f$ of $u, g$ of $v$ satisfy

$$
\Gamma(\alpha)(\operatorname{cls} f)=\operatorname{cls} g,
$$

then any bordered representative of $\alpha$ lifts to a homotopy $f \simeq g$.

To prove the first part of the proposition it suffices to define $\Gamma(\alpha)$ for all $u, v$ and $\alpha \in \pi A^{Z}(u, v)$, and then prove $\Gamma$ a functor. To define $\Gamma(\alpha)$, we choose a lift $f$ of $u$, and bordered representative $F$ of $\alpha$; then $F$ can be lifted to a homotopy $H$ of $f$, and if $g$ is the final map of $H$, we set

$$
\Gamma(\alpha)(\operatorname{cls} f)=\operatorname{cls} g \text {. }
$$

However it is necessary to prove $\Gamma(\alpha)$ is well-defined. There are several ways of doing this, but a way we like involves the notion of fibration of groupoids, 
which is defined in the exercises of [1], and which is treated in detail in [2]. So here we simply outline the method.

The first point is the following simple observation.

(2.2) Lemma. If $p: D \rightarrow A$ has the WCHP, then for any $Z$, the induced morphism $p_{*}: \pi D^{Z} \rightarrow \pi A^{Z}$ is a fibration of groupoids.

From a fibration of groupoids we obtain a functor:

(2.3) Lemma. If $p: G \rightarrow H$ is a fibration of groupoids, then there is a functor $\Gamma: H \rightarrow$ Set which on objects is given by

$$
\Gamma(u)=\pi_{0} p^{-1}(u) .
$$

Here $\pi_{0} p^{-1}(u)$ denotes the set of components of the groupoid $p^{-1}(u)$, the fibre over $u$. The point about this construction is that if $b \in H(u, v)$ and $f \in \in \pi_{0} p^{-1}(u)$, then the fibration property implies that $b$ lifts to an element $a \in G(f, g)$ for some $g$, and one defines $\Gamma(b)(\operatorname{cls} f)$ to be cls $g$. The proofs that $\Gamma(b)$ is well defined, and that $\Gamma$ is a functor, are simple.

In particular we can apply Lemma (2.3) to the fibration $p_{*}: \pi D^{Z} \rightarrow \pi A^{Z}$ when $p: D \rightarrow A$ has the WCHP. What has to be checked to prove the first part of Proposition (2.1) is:

(2.4) Lemma. If $p: D \rightarrow A$ has the WCHP, and $u: Z \rightarrow A$ is a map, then the sets $\pi_{0} p_{*}^{-1}(u)$ and $Z, D \backslash u$ coincide.

Proof. Let $f, g: Z \rightarrow D$ be lifts of $u$. Then $f \sim g$ will mean $f, g$ belong to the same components of $\pi_{0} p^{-1}(u)$, and this is true if and only if there is a homotopy $F: f \simeq g$ such that $p F$ is homotopic rel end maps to the stationary homotopy at $u$. Thus $f \simeq g$ rel $p$ implies $f \sim g$, and we have to prove the converse.

Let us assume $U: f \simeq g$ is a homotopy and $H: Z \times I \times I \rightarrow A$ is a homotopy rel $Z \times I$ of $p U$ to the stationary homotopy at $u$. Consider the diagram

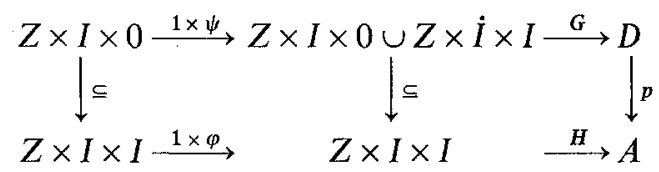

in which $G$ is defined by

$$
(z, s, t) \leadsto \begin{cases}U(z, s) & \text { if } t=0 \\ f(z) & \text { if } s=0 \\ g(z) & \text { if } s=1,\end{cases}
$$

$\varphi: I \times I \rightarrow I \times I$ is a homeomorphism which maps $I \times 0$ onto $I \times 0 \cup \dot{I} \times I$, and $\psi$ is the restriction of $\varphi$.

Now $H(1 \times \varphi)$ need not be bordered. However $H(1 \times \varphi)$ is homotopic rel end maps to a bordered homotopy $H^{\prime}$ and $H^{\prime}$ lifts to a homotopy $K$ of $p G(1 \times \psi)$. Then $L=K(1 \times \varphi)^{-1}$ is an extension of $G$ whose end map $(z, s) \leadsto$ $L(z, s, 1)$ is a homotopy $f \simeq g$ rel $p$ as required. 
We have now constructed the functor $\Gamma$ of Proposition (2.1). To prove the last part of Proposition (2.1), let $F$ be a bordered representative of $\alpha$, and suppose $F$ is stationary on $Z \times[s, 1](s<1)$. By an easy modification of the WCHP, $F \mid Z \times[0, s]$ lifts to a homotopy $H: f \simeq g^{\prime}$, say (of length $s$ ). By the construction of $\Gamma$, we have $g^{\prime} \simeq g$ rel $p$, by a homotopy $K$ which can be chosen of length $1-s$. Clearly $K$ and $H$ glue together to give a homotopy $f \simeq g$ which lifts $F$.

There are a number of consequences of Proposition (2.1). We still assume $p: D \rightarrow A$ has the WCHP.

(2.5) Proposition. If $\alpha \in \pi A^{Z}(u, v)$, then

$$
\Gamma(\alpha): Z, D \backslash u \rightarrow Z, D \rrbracket v
$$

is a bijection.

The proof is clear since $\Gamma$ is a functor from a groupoid.

This result generalises Lemma 2.1 of [5] which asserts (in our notation) that if $p$ has the CHP, $u \simeq v$, and $Z$ is a complex then $Z, D \backslash u$ is bijective with $Z, D \backslash v$.

Next let $f: W \rightarrow Z$ be a map. Then $f$ induces for each map $u: Z \rightarrow A$ a function

$$
f^{*}: Z, D \backslash u \rightarrow W, D \backslash u f
$$

(2.6) Proposition. If $W=Z$ and $f \simeq 1$, then $f^{*}$ is a bijection.

(2.7) Proposition. If $W=A$ and $f: A \rightarrow Z$ satisfies $u f \simeq 1$, then $f^{*}$ is a surjection.

(2.8) Proposition. If $f: W \rightarrow Z$ is a homotopy equivalence, then $f^{*}$ is a bijection.

The proofs of these propositions are of the types of those in [1] $\$ 7.2$ and so are left to the reader.

(2.9) Remark. It is interesting that for Lemmas 2.2, 2.4 we need only the RWCHP (rather weak CHP) which says that any homotopy $Z \times I \rightarrow A$ of $p f$ is homotopic $\operatorname{rel} Z \times \dot{I}$ to a homotopy which lifts to a homotopy of $f$. This property has been considered by Dold-Thom [4] ("relèvements des homotopies homotopes"). The interest of the RWCHP is simply that $p: D \rightarrow A$ has this property if and only if $p_{*}: \pi D^{Z} \rightarrow \pi A^{Z}$ is a fibration of groupoids for all $Z$. Further, the first part of Proposition (2.1) is valid for this weaker condition on $p$, but the last clause of Proposition (2.1) has then to be altered to: then some representative of $\alpha$ lifts to a homotopy $f \simeq g$. The RWCHP does not seem to be invariant under the formation of induced maps; however we do not know of maps which have the RWCHP but not the WCHP. The invariance property of the RWCHP is the following: Suppose $p: D \rightarrow A$ has the RWCHP, $q: E \rightarrow A$ is a map and $i: E \rightarrow D, r: D \rightarrow E$ are maps over $A$. If there is a homotopy $F: r i \simeq 1_{E}$ over a homotopy $G: 1_{A} \simeq 1_{A}$ such that $G$ is homotopic rel end maps to the stationary homotopy at $1_{A}$, then $q: E \rightarrow A$ has the RWCHP. However some of the later proofs break down if we assume the RWCHP rather than the WCHP. 


\section{Homotopy Inverses of Maps of Fibrations}

Suppose given a commutative diagram

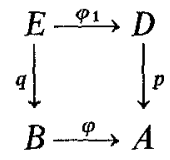

Then $\varphi$ and $\varphi_{1}$ are said to form a map $\varphi: q \rightarrow p$ over $\varphi$, and the idea of a homotopy $\varphi \simeq \psi$ of such maps over a homotopy $\varphi \simeq \psi$ is well-known. Our first object is to give conditions on $\varphi$ and $\varphi_{1}$ which ensure that $\varphi$ has a left-homotopy inverse. We shall assume throughout this section that $p$ and $q$ have the WCHP.

(3.2) Theorem. Let $\psi: A \rightarrow B$ be a left-homotopy inverse of $\varphi$, and $F: \psi \varphi \simeq 1$ a bordered homotopy. Suppose either (i) $\varphi_{1}$ is a homotopy equivalence, or (ii) $\varphi_{1}$ has a left-homotopy inverse $\psi_{1}$ such that $q \psi_{1}=\psi p$. Then $\varphi$ has a left-homotopy inverse; in fact there is a map $\psi: p \rightarrow q$ over $\psi$ and a homotopy $\psi \varphi \simeq 1$ over $F$.

Proof. We consider the diagram

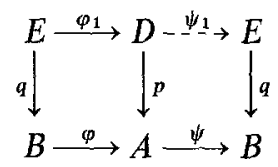

and part of our object is to construct a lifting $\psi_{1}$ of $\psi p$. Consider the functions

$$
D, E \backslash \psi p \stackrel{\varphi *}{\longrightarrow} E, E \backslash \psi \varphi q \stackrel{\Gamma(\alpha)}{\longrightarrow} E, E \backslash q
$$

where $\alpha$ is the class of the homotopy $F(q \times 1): E \times I \rightarrow B$.

If we know that $\varphi_{1}^{*}$ is a surjection, then we can choose a class $c$ in $D, E \backslash \psi p$ so that

$$
\Gamma(\alpha) \varphi_{1}^{*}(c)=\text { cls } 1 .
$$

If $\psi_{1}$ is a representative of $c$, then $\psi_{1}$ lifts $\psi p$ and, since $F$ is bordered, $F(q \times 1)$ lifts to a homotopy $\psi_{1} \varphi_{1} \simeq 1$. This would prove the result.

If $\varphi_{1}$ is a homotopy equivalence, then $\varphi_{1}^{*}$ is a bijection (Proposition (2.8)) and so a surjection. This settles case (i). In case (ii), we consider the diagram

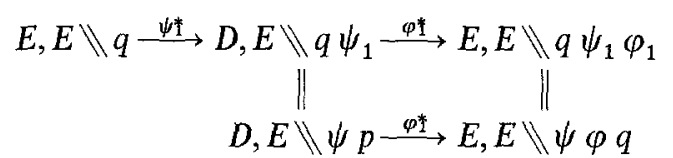

Since $\psi_{1} \varphi_{1} \simeq 1$, we have $\varphi_{1}^{*} \psi_{1}^{*}=\left(\psi_{1} \varphi_{1}\right)^{*}$ is a bijection, and so $\varphi_{1}^{*}$ is a surjection in this case also.

(3.3) Example. It is not true that $\varphi$ has a left-homotopy inverse if $\varphi$ and $\varphi_{1}$ both do so. As a counter example, let $\varphi$ be the map

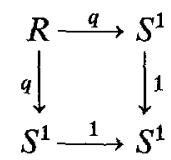


where $q$ is the covering map. Then $\varphi_{1}=q$ has a left-homotopy inverse because $R$ is contractible, and $\varphi=1$. However $\varphi$ cannot have a left-homotopy inverse, as is easily seen by considering 1-dimensional homology.

For the next result, we still refer back to diagram (3.1).

(3.4) Theorem. If $\varphi$ and $\varphi_{1}$ are homotopy equivalences, then so also is $\varphi$. In fact, let $\psi$ be any homotopy inverse of $\varphi$ and let $F: \psi \varphi \simeq 1, G: \varphi \psi \simeq 1$ be bordered homotopies. Then there is a map $\psi: p \rightarrow q$ over $\psi$, a homotopy $\psi \varphi \simeq 1$ over $F$, and a homotopy $\varphi \psi \simeq 1$ over the sum

$$
G+\varphi F(\psi \times 1)-G(\varphi \psi \times 1) .
$$

Proof. We apply (3.2) (i) to define $\psi$ and construct the homotopy $\psi \varphi \simeq 1$ over $F$. Then we again apply (3.2) (i) but with $\varphi$ replaced by $\psi$; we deduce that $\psi$ has a left-homotopy inverse $\varphi^{\prime}$ such that the homotopy $\varphi^{\prime} \psi \simeq 1$ covers $G$. The composite homotopy

$$
\varphi \psi \simeq \varphi^{\prime} \psi \varphi \psi \simeq \varphi^{\prime} \psi \simeq 1
$$

is then a homotopy as required.

(3.6) Corollary. Theorem 1.2 is true if $p, q, f, g$ all have the $W C H P$, and in this case the quadruple $\left(\Phi, \varphi_{1}, \varphi_{2}, \varphi\right)$ is a homotopy equivalence of squares.

Proof. We choose a homotopy inverse $\psi$ of $\varphi$ and we choose homotopies $F: \psi \varphi \simeq 1, G: \varphi \psi \simeq 1$. By Theorem (3.4) we can construct a homotopy inverse $\psi=\left(\psi_{1}, \psi\right)$ of $\boldsymbol{\varphi}=\left(\varphi_{1}, \varphi\right)$, and also a homotopy inverse $\psi^{\prime}=\left(\psi_{2}, \psi\right)$ of $\varphi^{\prime}=\left(\varphi_{2}, \varphi\right)$. Also the homotopies $\psi \varphi \simeq 1, \psi^{\prime} \varphi^{\prime} \simeq 1$ cover $F: \psi \varphi \simeq 1$, and the homotopies $\varphi \psi \simeq 1, \varphi^{\prime} \psi^{\prime} \simeq 1$ also cover the same homotopy $\varphi \psi \simeq 1$ (viz the homotopy given by (3.5)). The result follows.

Theorem (3.4) generalises Theorem (6.1) of [3]: Suppose in (3.1) that $B=A$, $\varphi=1$. Then $\varphi_{1}$ is said to be a fibre homotopy equivalence if there is a map $\psi_{1}$ : $D \rightarrow E$ over $1_{A}$, and there are homotopies $\psi_{1} \varphi_{1} \simeq 1, \varphi_{1} \psi_{1} \simeq 1$ over the stationary homotopy of $1_{A}$.

(3.7) Corollary (Dold). If in diagram (3.1), $\varphi=1$ and $\varphi_{1}$ is a homotopy equivalence, then $\varphi_{1}$ is a fibre homotopy equivalence.

Proof. In Theorem (3.4), let $\psi=1: A \rightarrow A$, and let $F, G$ be stationary homotopies. Then the homotopy in (3.5) is also stationary, so the corollary is a special case of Theorem (3.4).

(3.8) Remark. It seems difficult to better the curious "conjugate" homotopy (3.5) of Theorem (3.4). In particular, it is not always possible to find a map $\psi: p \rightarrow q$ over $\psi$ such that there are homotopies $\psi \varphi \simeq 1$ over $F$ and $\varphi \psi \simeq 1$ over $G$. As a counter-example, let $q=p$ be a covering map, and let $\varphi=1, \psi=1$. If $F: 1 \simeq 1$ is stationary, and $\psi_{1} \simeq 1$ is a homotopy over $F$, then $\psi_{1}$ must equal 1 . On the other hand suppose there is a homotopy $G: 1 \simeq 1: B \rightarrow B$ and an element $b \in B$ such that the loop $t \rightsquigarrow G(b, t)$ does not lift to a loop in $E$; then no homotopy $\psi_{1} \simeq 1$ can cover $G$. 
A fibration of pairs is a map $q:\left(E, E^{\prime}\right) \rightarrow\left(B, B^{\prime}\right)$ such that the map $E \rightarrow B$ determined by $q$ has the WCHP, and $E^{\prime}=q^{-1}\left(B^{\prime}\right)$. (This implies, of course, that $E^{\prime} \rightarrow B^{\prime}$ has the WCHP.) Suppose now given a map of fibrations of pairs

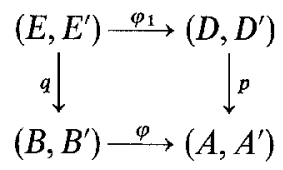

(3.9) Corollary. If $\varphi:\left(B, B^{\prime}\right) \rightarrow\left(A, A^{\prime}\right)$ and $\varphi_{1}: E \rightarrow D$ are homotopy equivalences, then $\varphi_{1}:\left(E, E^{\prime}\right) \rightarrow\left(D, D^{\prime}\right)$ is a homotopy equivalence of pairs.

Proof. It is straightforward to check that if we choose $\psi$ and $F: \psi \varphi \simeq 1$, $G: \varphi \psi \simeq 1$ so that $\psi$ and $F, G$ are maps and bordered homotopies of pairs then the map $\psi_{1}$ and the homotopies $\psi_{1} \varphi_{1} \simeq 1, \varphi_{1} \psi_{1} \simeq 1$ given by Theorem (3.4) are also maps and homotopies of pairs.

(There is an easy generalisation of this to $M$-ads.)

It is convenient to place the following corollary in this section, because it is in the same circle of ideas, although its proof uses (1.4) which is a corollary of the coglueing theorem proved in the next section.

(3.10) Corollary. Let $p:\left(D, D^{\prime}\right) \rightarrow\left(A, A^{\prime}\right)$ be a fibration and let $\varphi:\left(B, B^{\prime}\right) \rightarrow$ $\left(A, A^{\prime}\right)$ be a homotopy equivalence. Then $\varphi_{1}:\left(\varphi^{*}(D), \varphi^{*}\left(D^{\prime}\right)\right) \rightarrow\left(D, D^{\prime}\right)$ is a homotopy equivalence.

Proof. By Corollary (1.4), $\varphi_{1}: \varphi^{*}(D) \rightarrow D$ is a homotopy equivalence. So the result follows from Corollary (3.9).

Corollary (3.10) seems to be assumed in the proof of Lemma 9.2 .2 of $[8]^{1}$ although the proof there can be carried through by using homology and the 5-lemma.

One result we have not been able to prove or disprove is the following: if $p: D \rightarrow A$ has the WCHP and $B$ is a strong deformation retract of $A$, then $E=p^{-1}(B)$ is a strong deformation retract of $D$. This seems to be assumed in the proof of Corollary 8.5.4 of $[8]^{1}$ although here again the proof can be carried through either by a homology argument or by the following result, which is convenient to place here even though the only result used from this paper is Corollary (1.4).

Let $p: D \rightarrow A$ have the CHP, let $B$ be a closed subset of $A$ such that $(A, B)$ has the HEP, let $E=p^{-1}(B)$ and let $q: E \rightarrow B$ be the restriction.

(3.11) Proposition. If the inclusion $B \rightarrow A$ is a homotopy equivalence, then $q$ is a strong deformation retract of $p$.

Proof. By 7.4.1 (Corollary 2) of [1], there is a retracting homotopy $R: A \times I \rightarrow A$ which is stationary on $B$ and retracts $A$ onto $B$. Let $F: D \times 0 \cup E \times$ $I \rightarrow D$ be the identity on $D$ and stationary on $E$. By Theorem 4 of [9] $R(p \times 1): D \times I \rightarrow A$ lifts to a homotopy $H: D \times I \rightarrow D$ which extends $F$. Then $H$ and $R$ define the required homotopy $p \times I \rightarrow p$ retracting $p$ onto $q$.

${ }^{1}$ We are grateful to R. M. F. Moss for these observations on [8]. 


\section{The Double Mapping Track}

In order to deduce Theorem (1.2) from Corollary (3.6) we need to replace the maps $f, g$ in diagram (1.1) by fibrations. This process is well known but needs, for our purposes, to be clarified.

Since $(I, \dot{I})$ has the HEP, the restriction map $B^{I} \rightarrow B^{i}$ is a Hurewicz fibration. It follows, using the obvious homeomorphism $B^{\dot{I}} \rightarrow B \times B$, that the map

$$
\theta: B^{I} \rightarrow B \times B \quad \lambda \leadsto(\lambda(0), \lambda(1))
$$

is a Hurewicz fibration.

Let $g: Y \rightarrow B, q: E \rightarrow B$ be maps. Their double mapping track is $P(g, q)$ defined by the pull-back

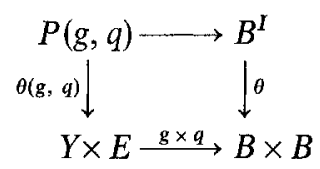

Notice that $\theta(g, q)$ also is a Hurewicz fibration. In terms of elements $P(g, q)$ can be taken as the subset of $Y \times E \times B^{I}$ of elements $(y, e, \lambda)$ such that $g(y)=\lambda(0)$, $q(e)=\lambda(1)$. So there is a homeomorphism

$$
P(g, q) \rightarrow P(q, g) \quad(y, e, \lambda) \rightsquigarrow(e, y,-\lambda) .
$$

For $i=0,1$, let $\varepsilon_{i}: B^{I} \rightarrow B$ be the evaluation mapping $\lambda \leadsto \lambda(i)$.

Let $P_{i}(q)$ be defined by the pull-back

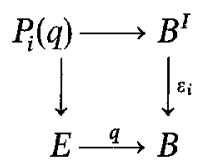

and let $q_{i}: P_{i}(q) \rightarrow B$ be the mapping $(e, \lambda) \leadsto \lambda(1-i)$. Then we have homeomorphisms

$$
\begin{array}{ll}
\xi_{0}: P_{0}(q) \rightarrow P(q, 1), & \xi_{1}: P_{1}(q) \rightarrow P(1, q), \\
(e, \lambda) \rightsquigarrow(e, \lambda(1), \lambda), & (e, \lambda) \rightsquigarrow(\lambda(0), e, \lambda) .
\end{array}
$$

It follows easily that $q_{0}: P_{0}(q) \rightarrow P(q, 1)$ is the composite

$$
P_{0}(q) \stackrel{\xi_{0}}{\longrightarrow} P(q, 1) \stackrel{\theta(\xi, 1)}{\longrightarrow} E \times B \stackrel{\text { Proj. }_{\longrightarrow}}{\longrightarrow} B .
$$

Hence $q_{0}$ is the composite of Hurewicz fibrations; so $q_{0}$, and similarly $q_{1}$, is a Hurewicz fibration (as is well-known).

(4.1) Lemma. There are pull-back squares

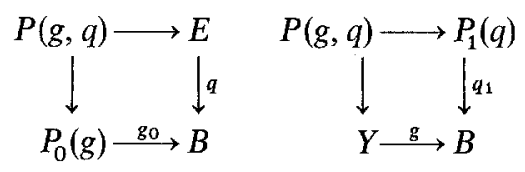


This is obvious from the explicit formula for $P(g, q)$.

Let $k: E \rightarrow P_{i}(q)$ be the map $e \leadsto(e, \hat{e})$ where $\hat{e}$ is the constant path at $e$. Then we have a commutative diagram

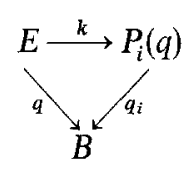

It is well-known that $k$ is a homotopy equivalence.

Suppose further that we have a pull-back square

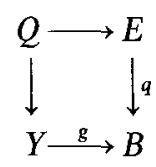

(4.2) Proposition. If $q$ has the WCHP, then the map

$$
\bar{k}: Q \rightarrow P(g, q), \quad(y, e) \rightarrow(y, e, \hat{e})
$$

is a homotopy equivalence.

Proof. The map $\bar{k}$ is induced (or pulled-back) by $g$ from $k: E \rightarrow P_{1}(q)$. But $q_{1} k=q$, and $k$ is a homotopy equivalence. By Corollary (3.7), $k$ is a fibre homotopy equivalence. But the pull-back of a fibre homotopy equivalence is a fibre homotopy equivalence, and a fortiori is a homotopy equivalence.

\section{Proof of the Coglueing Theorem}

We now go back to the situation of diagram (1.1).

The constructions $P(g, q), P_{1}(q), P_{0}(g)$ of the previous section are functorial in $g$ and $q$. So the maps $\varphi, \varphi_{1}, \varphi_{2}$ induce a commutative cube

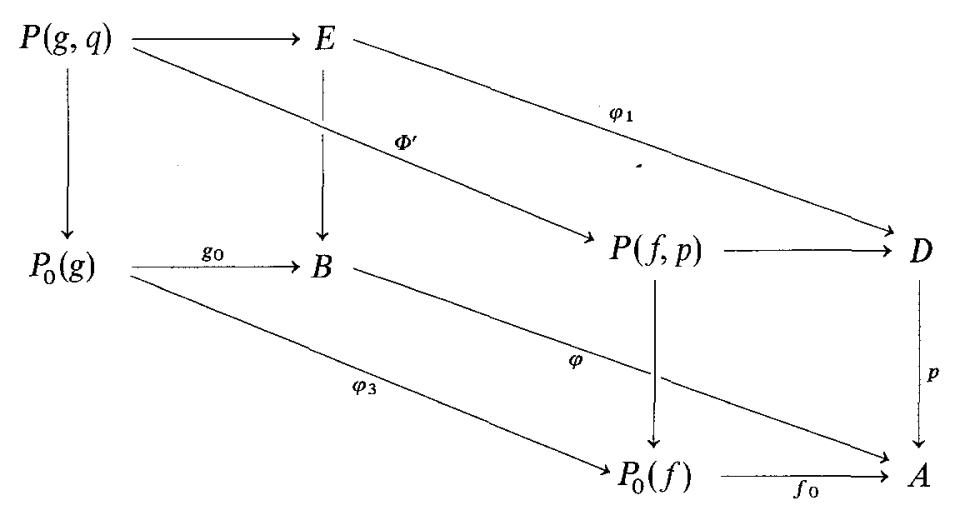


The vertical maps $k$ in the following commutative diagram, and also $\varphi_{2}$, are homotopy equivalences.

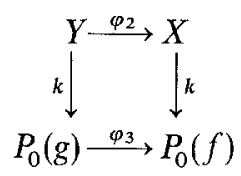

It follows that $\varphi_{3}$ is a homotopy equivalence. So Corollary (3.6), applied to (5.1) instead of (1.1), shows that $\Phi^{\prime}$ is a homotopy equivalence.

However the following diagram is commutative.

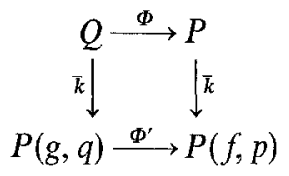

By Proposition (4.2), the vertical maps $\bar{k}$ are homotopy equivalences. It follows that $\Phi$ is a homotopy equivalence, and this is Theorem (1.2).

\section{The Spectral Sequence}

This section is an addendum to the paper of Dress [5].

Let $q: E \rightarrow B$ be a map, and let $S(B)$ be the singular complex of $B$. A local system $T_{r}$ from $S(B)$ to abelian groups is defined as follows: Let $\Delta(n)$ denote the standard $n$-simplex and let $\sigma \in S_{n}(B)$. Then $\sigma \in B^{\Delta(n)}$. Let $F_{\sigma}$ be the fibre over $\sigma$ of the induced map $E^{\Delta(n)} \rightarrow B^{\Delta(n)}$. Then we set

$$
T_{r}(\sigma)=H_{r}\left(F_{\sigma}\right) .
$$

Now let $\varepsilon: \Delta(n) \rightarrow \Delta(m)$ be a simplicial map preserving the order of the vertices. Then $\varepsilon$ induces a commutative diagram

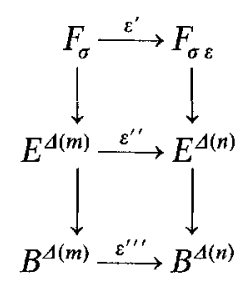

and we define $T_{r}(\varepsilon)$ to be the map of homology induced by $\varepsilon^{\prime}$.

According to [5] the crucial step in constructing the Serre spectral sequence is proving that $T_{r}$ is stable, i. e. that $T_{r}(\varepsilon)$ is an isomorphism for all $\varepsilon$.

Suppose that $q$ has the WCHP. Then so also do the induced map: $E^{\Delta(n)} \rightarrow$ $B^{\Delta(n)}$ for all $n$. Now $\varepsilon: \Delta(n) \rightarrow \Delta(m)$ is a homotopy equivalence. So in (6.1), $\varepsilon^{\prime \prime}, \varepsilon^{\prime \prime \prime}$ are homotopy equivalences. By Corollary (1.5), $\varepsilon^{\prime}$ is a homotopy equivalence, and so $T_{r}\left(\varepsilon^{\prime}\right)$ is an isomorphism. 


\section{Further Problems}

(7.1) One obvious problem is to generalise the results here to inverse limits. Some assumptions on the diagrams over which the limits are taken would probably be required; in some simple cases one can obtain results by repeated applications of those of this paper.

(7.2) An open problem is whether the results of this paper are true if the words "homotopy equivalences" are replaced by "simple homotopy equivalences" (one must assume for this that all spaces are $\mathrm{CW}$-complexes). There is a similar problem in the dual case, which boils down to deciding if the Whitehead group of a free product with amalgamation is generated by the images of the Whitehead groups of the factors.

\section{References}

1. Brown, R.: Elements of modern topology. Maidenhead: McGraw-Hill 1968.

2. - Fibrations of groupoids. J. Algebra (to appear).

3. Dold, A.: Partitions of unity in the theory of fibrations. Ann. of Math. 78, 223-255 (1963).

4. - Thom, R.: Une géneralisation de la notion d'espace fibré. Application aux produits symetriques infinis. C. R. Acad. Sci. Paris 242, 1680-1682 (1956).

5. Dress, A.: Zur Spektralsequenz von Faserungen. Inventiones Math. 3, 172-178 (1967).

5 a. Heath, P. R.: Induced homotopy equivalences on mapping spaces and duality (to appear).

6. James, I. M., Thomas, E.: Note on the classification of cross-sections. Topology 4, 351-359 (1966).

7. Weinzweig, A. I.: Fibre spaces and fibre homotopy equivalences. Colloqu. on Algebraic Topology, Aarhus (1962).

8. Spanier, E. H.: Algebraic topology. New York: McGraw-Hill 1966.

9. Str $\phi \mathrm{m}$, A.: Note on cofibrations. Math. Scandinav. 19, 11-14 (1966).

\section{P.R. Heath}

Department of Mathematics

Memorial University

St. Johns, Newfoundland

Canada
Dr. Ronald Brown

Dept. of Pure Mathematics

The University of Hull

22-24 Newland Park

Hull, Great Britain

(Received July 25, 1969) 